\title{
Review
}

\section{Transcriptional regulation of secondary wall formation controlled by NAC domain proteins}

\author{
Masatoshi Yamaguchi ${ }^{1,2}$, Taku Demura ${ }^{1,2,3, *}$ \\ ${ }^{1}$ Graduate School of Biological Sciences, Nara Institute of Science and Technology, Ikoma, Nara 630-0192, Japan; \\ ${ }^{2}$ RIKEN Plant Science Center, Yokohama, Kanagawa 230-0045, Japan; ${ }^{3}$ RIKEN Biomass Engineering Program, \\ Yokohama, Kanagawa 230-0045, Japan \\ *E-mail: demura@bs.naist.jp Tel: +81-743-72-5460 Fax: +81-743-72-5469
}

Received September 9, 2009; accepted April 21, 2010 (Edited by N. Mitsuda)

\begin{abstract}
Woody cells develop secondary wall structure that mainly consists of polysaccharides (cellulose and hemicellulose) and lignin. These components are expected to be new sources of biofuels and biomaterials. Therefore, it is important to understand the molecular mechanism underlying secondary wall formation and how it contributes to plant biomass. Plant-specific NAC domain transcription factor family has been shown to be involved in diverse biological functions. Recently, several studies reported that a subfamily of the NAC domain transcription factors plays pivotal roles in secondary wall formation. In this review, we have summarized the role of NAC domain transcription factors in controlling the secondary wall formation.
\end{abstract}

Key words: Fiber cell, NAC domain protein, secondary wall, transcription factor, xylem vessel.

The plant vascular tissue consists of phloem and xylem. Phloem transports nutrients such as amino acids and sucrose. Xylem functions in the conduction of water and minerals throughout the plant and also supports the plant body. One of the characteristic features of xylem cells is a secondary wall structure between plasma membrane and (primary) cell wall. Studies on differentiation of xylem cells have been considered a good model system for the analysis of cell differentiation in higher plants because there are several well-established in vitro induction systems, in which isolated cells or suspension cultured cells from various plants transdifferentiate into xylem cells (reviewed in Fukuda 1996, 2004; Turner et al. 2007). Recent interest in biofuels has raised the possibility that a better understanding of xylem development can be utilized for the improvement of plant biomass, since major portions of wood, which represents one of important sources of woody biomass, is mainly composed of two types of xylem cells, xylem vessels and fiber cells. Moreover, main components of the secondary wall are polysaccharides, cellulose, and hemicellulose, which are expected to be good starting materials for the production of bioethanol and bioplastics.

Transcription factors are proteins that have function in controlling the expression of target genes quantitatively, temporally, and spatially. To date, genetic analyses have revealed a number of transcription factors regulating vascular development (reviewed in Ariel et al. 2007; Carlsbecker and Helariutta 2005; Demura and Fukuda 2007). Moreover, reverse genetic approaches have been successful in isolating several NAC (which stands for NAM, ATAF1/2 and CUC2) domain transcription factors that control the specification of xylem cells accompanied by secondary wall formation. In this review, we summarize the function and regulation of the NAC domain proteins controlling secondary wall formation.

VND/NST/SND1 subfamily of NAC domain proteins regulate secondary wall formation

Previously, we established in vitro transdifferentiation systems, in which zinnia mesophyll cells and Arabidopsis suspension cells could synchronously transdifferentiate into tracheary elements at a high frequency. By using microarray analysis we identified a number of genes whose expression is elevated during the transdifferentiation processes (Demura et al. 2002; Kubo et al. 2005), including the following genes belonging to a subfamily of the NAC transcription factor family: zinnia Ze567 and Arabidopsis VND1 to VND7 (Figure 1).

Abbreviations; ANAC012, arabidopsis nac domain containing protein012; ASL, asymmetric leaves2-like; BRN, bearskin; KNAT7, knotted1-like homeodomain protein 7; LBD, lateral organ boundaries domain; NAC, NAM, ataf1/2 and cuc2; NST, nac secondary wall thickening promoting factor; SMB, sombrero; SND, secondary wall-associated nac domain protein; VND, vascular-related nac-domain protein; VNI, vnd-interacting; XND1, xylem nac domain 1

This article can be found at http://www.jspcmb.jp/ 


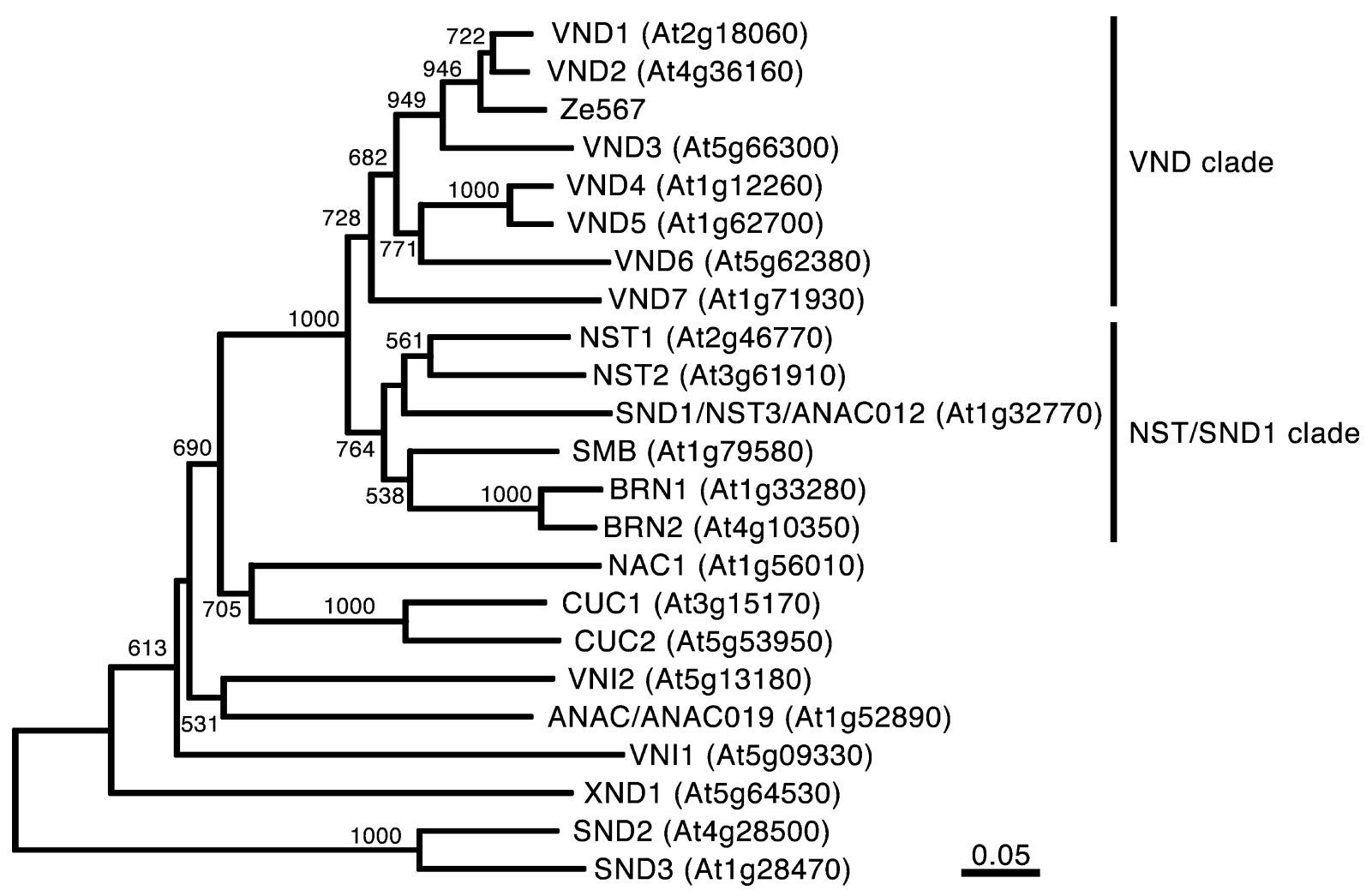

Figure 1. Phylogenetic tree of NAC domain proteins including members of the VND and NST/SND1 clades. Ze567 is isolated as a gene specifically expressed during in vitro differentiation into tracheary elements in Zinnia elegans (Demura et al. 2002). AGI code of Arabidopsis NAC domain proteins are indicated in parentheses. A tree was constructed using the neighbor-joining method with ClustalX. Bootstrap values above 500 out of 1,000 replicates were indicated.

Expression analysis revealed that all $V N D$ genes are preferentially expressed in developing vascular cells of roots: $V N D 1, V N D 2$, and $V N D 3$ are expressed in procambial cells; VND4 and VND5 in the differentiating vessels; VND6 specifically in the inner-metaxylem vessels; and VND7 in the protoxylem poles of procambium region as well as in differentiating both protoxylem and metaxylem vessels (Kubo et al. 2005; Yamaguchi et al. 2008). When we ectopically expressed the $V N D$ genes under the control of the cauliflower mosaic virus $35 \mathrm{~S}$ promoter (35Spro), 35Spro: VND6 and 35Spro: VND7 plants exhibited transdifferentiation of various types of cells into xylem vessel elements (Kubo et al. 2005). Interestingly, morphology of the transdifferentiated xylem vessel elements in roots of 35Spro:VND6 and 35Spro:VND7 plants was clearly different: VND6 induced xylem vessel elements with reticulated or pitted pattern of secondary wall similar to that of metaxylem vessels while VND7 induced xylem vessel elements with annular or spiral pattern of secondary wall which is typical of protoxylem vessels (Kubo et al. 2005). These data demonstrate that VND6 and VND7 can act as key regulators of differentiation of two different types of xylem vessels. Moreover, overexpression of these Arabidopsis VND6 and VND7 genes in poplar leaves also induces transdifferentiation of mesophyll and epidermal cells into metaxylem- and protoxylem-like vessel elements, respectively (Kubo et al. 2005), suggesting that molecular mechanism of xylem vessel differentiation is at least partially conserved between Arabidopsis and poplar. Loss-offunction phenotypes of each $V N D$ gene do not show any detectable defects in morphology (Kubo et al. 2005). However, transgenic plants expressing the dominant negative forms of VND7 driven by its own promoter exhibited a dwarf phenotype in the aerial parts with inhibition of protoxylem and metaxylem vessel formation in roots and of continuous vessel formation in the aerial parts (Yamaguchi et al. 2008). These results strongly suggest that VND7 plays a critical role in the formation of all types of vessels, together with other VND proteins functioning synergistically and/or redundantly during xylem vessel differentiation. To evaluate this hypothesis, it would be important to analyze multiple mutant combinations of $V N D$ genes.

NAC SECONDARY WALL THICKENING PROMOTING FACTOR1 (NST1), NST2, and SECONDARY WALL-ASSOCIATED NAC DOMAIN PROTEIN 1 (SND1)/NST3/ARABIDOPSIS NAC DOMAIN CONTAINING PROTEIN012 (ANAC012) belong to the same subfamily that contains the $V N D$ genes (Figure 1; Ko et al. 2007; Mitsuda et al. 2005, 
2007; Ooka et al. 2003; Zhong et al. 2006, 2007b). Overexpression of NST1, NST2, and SND1/NST3/ANAC012 induced ectopic lignified secondary cell wall thickenings in various tissues, phenocopy the overexpression of VND6 and VND7 (Ko et al. 2007; Mitsuda et al. 2005, 2007; Zhong et al. 2006). Expression analyses showed that NST2 is expressed in anther endothecium (Mitsuda et al. 2005), SND1/NST3/ANAC012 is expressed in fiber cells of inflorescence stems and hypocotyls and the valve endocarp layer and cells surrounding vascular vessels in the replum of siliques (Ko et al. 2007; Mitsuda et al. 2007; Mitsuda and Ohme-Takagi 2008; Zhong et al. 2006), and NST1 expression overlaps with both NST2 and SND1/NST3/ANAC012 gene expression patterns (Mitsuda et al. 2007; Mitsuda and Ohme-Takagi 2008). Single knockout of each gene does not exhibit a clear phenotype, with the notable exception of nst 1 mutant plants, which lose secondary wall formation in valve margin of siliques (Mitsuda and Ohme-Takagi 2008). Double knockouts of the nst 1 and nst 2 or nst 1 and snd1/nst3/anac012 loci showed defects in secondary cell wall formation of anther cells or fiber cells and valve margin and endocarp cells in siliques, respectively (Mitsuda et al. 2007; Mitsuda and Ohme-Takagi 2008; Zhong et al. 2007b). These results indicated that NST1 and SND1/NST3/ANAC012 also function in secondary wall formation of various tissues except xylem vessels.

A recent report has shown that sombrero $(\mathrm{smb})$ mutation increases root cap cell layers and that a gene encoding an NAC domain protein (At1g79580) which belongs to the NST/SND1 clade (Figure 1) is the causal gene of the smb mutation (Willemsen et al. 2008). Interestingly, overexpression of SMB and two remaining members in the NST/SND1 clade, BEARSKIN1 (BRN1, At1g33280/ANAC015) and BRN2 (At4g10350/ ANAC070), resulted in the ectopic secondary wall formation of various types of cells (Bennett et al. 2010). Since $S M B, B R N 1$, and $B R N 2$ are strongly expressed in root cap cells and the smb vrn1 vrn2 triple mutant showed defects in maturation of the root cap but not in xylem development (Bennett et al. 2010; Willemsen et al. 2008), it is suggested that SMB, BRN1, and BRN2 regulate root cap development rather than secondary wall formation even if they can mimic the function of VND and NST proteins.

\section{Domain structure}

NAC domain protein family contains conserved sequences, known as the "NAC domain", in the Nterminal region. In contrast, the C-terminal parts of NAC domain proteins are highly diverse and do not contain any known protein domains (Review in Olsen et al. 2005). The NAC domain proteins in the VND/NST/SND1 subfamily contain two highly

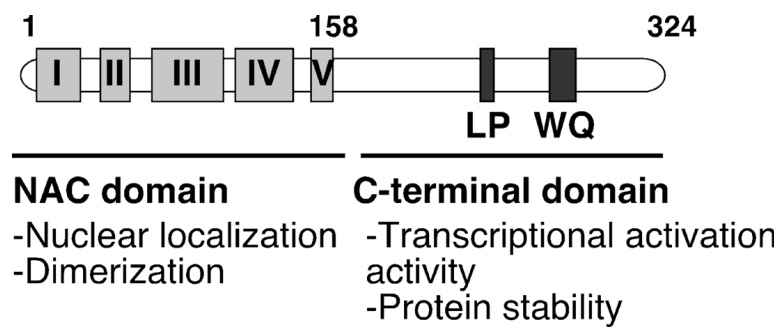

Figure 2. Schematic diagram of the domain structure of VND7 protein. VND7 protein has NAC domains consisting of 5 sub-domains, I to $\mathrm{V}$, at the $\mathrm{N}$ terminus. The NAC domain of the VND7 protein plays important roles in nuclear localization and dimerization with VND and VNI proteins. C-terminal region of the VND7 protein, which contains LP- and WQ-motifs, regulates transcriptional activation and is involved in protein stability.

conserved motifs, the LP-box and the WQ-box, in the Cterminal regions (Ko et al. 2007). Analysis of both yeast and plant cells have demonstrated that NAC domain of VND7 is required for nuclear localization and homo- or hetero-dimerization, while the C-terminal regions of VND7 and SND1/NST3/ANAC012 regulate transcriptional activation (Figure 2; Ko et al. 2007; Yamaguchi et al. 2008; Zhong et al. 2006). Observations of transgenic plants overexpressing the full length or Cterminally truncated VND7 under the control of $35 \mathrm{~S}$ promoter indicate that the entire $\mathrm{C}$-terminal region of VND7 is required for inducing vessel transdifferentiation. Interestingly, overexpression of truncated VND7 proteins lacking both LP-box and the WQbox resulted in discontinuous formation of one or both protoxylem vessel columns, suggesting that the C-terminally truncated VND7 proteins exert a dominant negative effect on xylem vessel formation (Yamaguchi et al. 2008). The C-terminally truncated VND7 protein is shown to be more stable than the full length VND7 protein, suggesting that this region is involved in VND7 protein stability (Yamaguchi et al. 2008).

\section{Downstream targets of VND and NST proteins}

As described above, overexpression of VND6, VND7, and NST genes can induce the ectopic secondary wall formation of various types of cells. Expression analyses demonstrate that VND7 and NSTS upregulate a number of genes previously shown to be associated with pathways required for secondary wall formation such as biosynthesis of cellulose, hemicellulose, and lignin (Kubo et al. 2005; Mitsuda et al. 2005; Yamaguchi et al. 2010a, 2010b; Zhong et al. 2006, 2007b). However, genes involved in programmed cell death during xylem vessel differentiation were induced by the overexpression of VND7 (Kubo et al. 2005; Yamaguchi et al. 2010a, 2010b), but not that of NST genes (Mitsuda et al. 2005; Zhong et al. 2006). It is known that xylem vessels undergo secondary wall thickening followed by programmed cell death, whereas fibers produce 


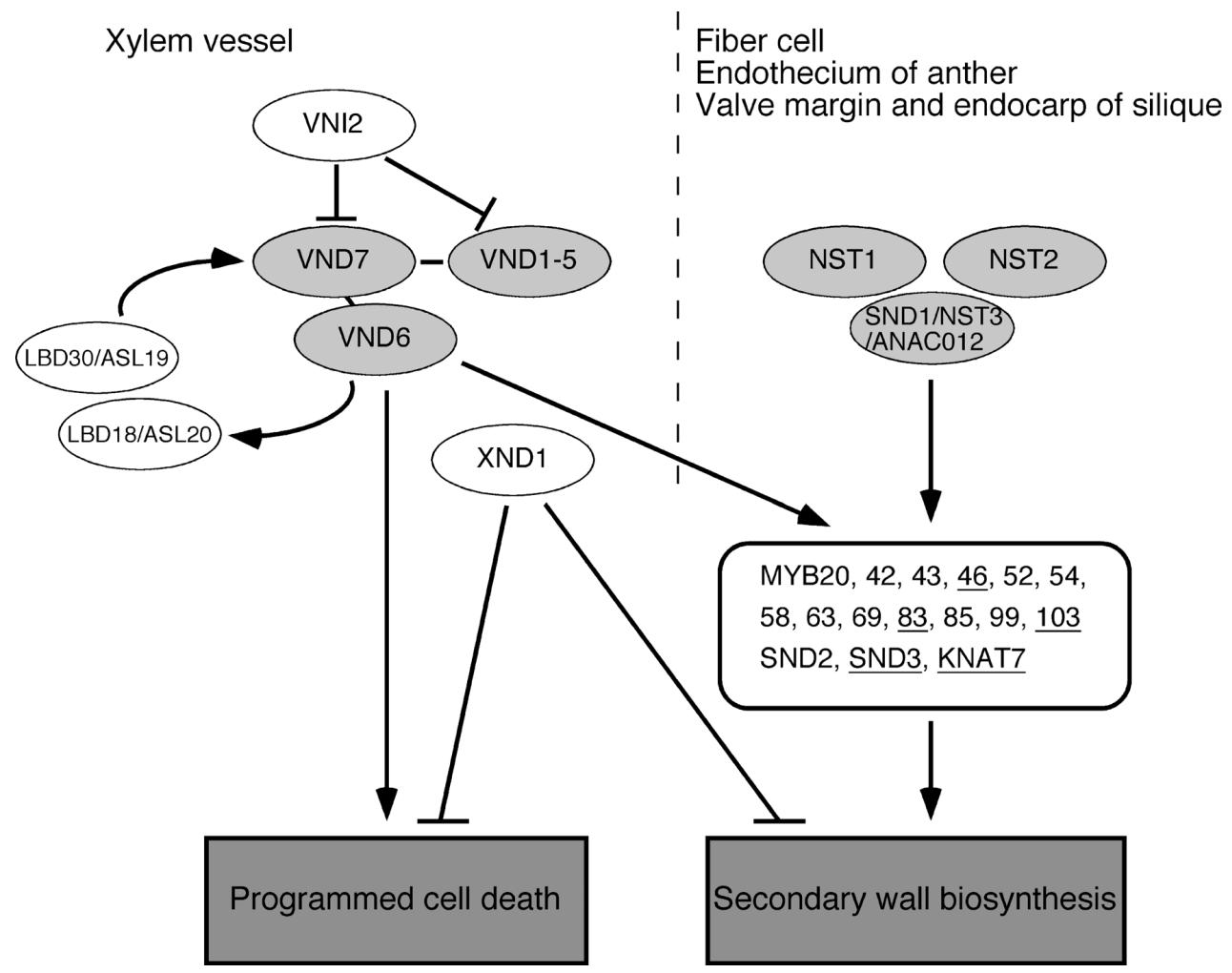

Figure 3. Schematic diagram of the transcriptional network regulating secondary wall formation and programmed cell death. Members of the VND and NST/SND1 clades of the NAC domain proteins control the gene expression of the transcription factors that regulate the genes involved in secondary wall formation. VND proteins and XND1 protein positively and negatively regulate genes expression involved in programmed cell death as well as secondary wall formation, respectively. Transcription factors shown with underline are direct targets of the VND and NST proteins.

secondary walls without immediate cell death. The previous microarray data indicate that VND7 (and probably other VND proteins) controls the expression of genes involved in both secondary wall formation and programmed cell death required for xylem vessel differentiation while NST proteins upregulate expression of genes associated with secondary wall formation but not cell death (Figure 3; Mitsuda et al. 2005; Yamaguchi et al. 2010). How these two clades differentially regulated the expression of same or different genes is an important area of investigation.

It has been reported that VND/NST/SND1 as well as a number of other transcription factors such as other NAC domain proteins, MYB proteins, and homeobox proteins, are highly expressed in xylem tissues. Recently several studies have been reported that some of these transcription factors are downstream of the VND/NST/SND1 (Ko et al. 2009; McCarthy et al. 2009; Yamaguchi et al. 2010a, 2010; Zhong et al. 2007a, 2008; Zhou et al. 2009). Particularly, MYB46, MYB83, MYB103, and KNOTTED1-LIKE HOMEODOMAIN PROTEIN 7 (KNAT7) are direct targets of SND1/NST3/ANAC012 and possibly of NST1, VND6, and VND7 (Figure 3; Ko et al. 2009; McCarthy et al. 2009; Zhong et al. 2007a). Overexpression of MYB46 or MYB83 activates gene expression of biosynthetic pathways of cellulose, hemicellulose and lignin and induces ectopic secondary wall formation (Ko et al. 2009; McCarthy et al. 2009; Zhong et al. 2007a). Double mutation of MYB46 and MYB83 causes severe dwarf phenotype (McCarthy et al. 2009; N. Nishikubo, Y. Nakano, T.D. unpublished data). These data suggest existence of a transcriptional network regulating secondary wall formation (Figure 3 ). In addition to the MYB46 and MYB83, microarray analysis reveals that 8 other $M Y B$ genes are transiently expressed during the process of transdifferentiation into tracheary elements (Kubo et al. 2005; Nakano et al. 2010 in this issue). It is interesting to understand how these $M Y B$ genes form transcription network. MYB99 is specifically expressed in xylem vessels but not interfascicular fiber cells in inflorescence stems (Nakano et al. 2010 in this issue), suggesting that MYB99 genes might play some specific roles in xylem vessel differentiation.

Soyano et al. (2008) reported that two members of LATERAL ORGAN BOUNDARIES DOMAIN (LBD)/ASYMMETRIC LEAVES2 (AS2) transcription factor family regulate xylem vessel differentiation. Expression of LBD18/AS2-LIKE2O (ASL2O) and LBD30/ASL19 genes are observed in xylem vessels and are upregulated by VND6 and VND7. Interestingly, overexpression of LBD18/ASL2O and LBD30/ASL19 
genes induce transdifferentiation of cells from nonvascular tissues into xylem vessels, and ectopic expression of VND6 and VND7 genes. Therefore, the authors concluded that LBD18/ASL20 and LBD30/ASL19 genes appear to be involved in a positive feedback loop for $V N D 6$ and VND7 expression (Figure 3).

\section{Post-translational regulations of VND proteins}

Recent analysis demonstrated that the function of the NAC domain proteins is controlled at the posttranslational level (reviewed in Olsen et al. 2005). Indeed, VND7 forms either a homodimer or heterodimer with other VND proteins (Yamaguchi et al. 2008). Recently, we screened for proteins that interact with VND7 with a yeast two-hybrid system, and identified cDNAs encoding NAC domain proteins, VNDINTERACTING1 (VNI1) and VNI2 (Yamaguchi et al. 2010 b). In vitro pull-down assay demonstrated that VNI2 protein can effectively bind to VND1, VND2, VND3, VND4, and VND5 proteins as well as VND7 protein. The expression of VNI2 partially overlaps with that of VND7 in vessel precursors in roots, whereas the initiation and termination of VNI2 expression always precedes those of VND7 expression. Transient reporter assays showed that VNI2 is a transcriptional repressor and can repress the expression of vessel-specific genes regulated by VND7. The expression of VNI2 under the control of the VND7 promoter resulted in the inhibition of the normal development of xylem vessels in roots and aerial organs (Yamaguchi et al. 2010b). These data suggest that VNI2 regulates xylem cell specification as a transcriptional repressor that interacts with VND7.

The stability of VND7 could be regulated by proteasome-mediated degradation (Yamaguchi et al. 2008) and VND7 appears to be phosphorylated (M.Y., T.D. unpublished data). These data suggest that VND (and also NST) proteins might be controlled by several interacting partners.

\section{Other NAC domain proteins involved in secondary wall formation}

XYLEM NAC DOMAIN 1 (XND1) was isolated as a gene highly expressed in xylem (Zhao et al. 2005). Overexpression of XND1 resulted in dwarfing and suppression of xylem vessel differentiation (Zhao et al. 2008). Observation of electron microscopy demonstrated that overexpression of XND1 inhibited secondary wall deposition and autolysis in xylem vessels. Moreover, transgenic plants overexpressing XND1 fused to a repressor domain ( $X N D 1-S R D X)$ phenocopied gain-of function plants (Zhao et al. 2008), suggesting that XND1 is a transcriptional repressor consequently regulating expression of genes involved in both programmed cell death and secondary wall formation.

Zhong et al. (2008) reported that two NAC domain proteins, SND2 and SND3, are highly expressed in xylem and interfascicular fiber cells but not in pith of inflorescence stems, which are expressed downstream of SND1/NST3/ANAC012 (Figure 3). Overexpression of SND2 and SND3 increases secondary wall thickness of interfascicular fibers and xylary fibers, and induces the expression of a cellulose synthase (CesA8) gene (Zhong et al. 2008), suggesting that other NAC domain proteins also participate in secondary cell wall formation.

\section{Perspectives}

Here, we have reviewed recent findings on the NAC domain proteins belonging to the VND/NST/SND1 subfamily and their role in the secondary wall formation. However, further analysis is needed to better understand how secondary wall formation is regulated. For example, how the expression of genes in the VND/NST/SND1 subfamily itself is regulated is currently poorly understood. It is also important to identify the consensus DNA sequences recognized by members of the VND/NST/SND1 subfamily, and to analyze the loss-offunction mutants of $V N D$ genes.

Recently, characterization of NAC domain proteins closely related to the VND/NST/SND1 subfamily of poplar was reported (Zhong et al. 2010). Expression of these genes restored the secondary wall defects in Arabidopsis nst 1 and snd1/nst3/anac012 double mutant (Zhong et al. 2010) suggesting that further analyses of these genes could leads to understanding of the regulatory mechanism of wood formation in trees. Moreover, we have shown that expression of VND7 under the control of a chemically inducible system led to vessel differentiation in tobacco BY-2 cultured cells (Yamaguchi et al. 2008, 2010a). These transgenic cells are invaluable tools for biochemical analysis. We expect that the results obtained through these experiments will lead to the improvement of plant biomass production near in the future.

\section{Acknowledgements}

We would like to thank all of our coworkers. This work was supported by Grant-in-Aid for Scientific Research from the Ministry of Education, Culture, Sports, Science and Technology of Japan (grant no. 20770041, MY and no. 21027031 and 22370020, TD).

\section{References}

Ariel FD, Manavella PA, Dezar CA, Chan RL (2007) The true story of the HD-Zip family. Trends Plant Sci 9: 419-426

Bennett T, van den Toorn A, Sanchez-Perez GF, Campilho A, Willemsen V, Snel B, Scheres B (2010) SOMBRERO, BEARSKIN1, and BEARSKIN2 regulate root cap maturation in Arabidopsis. Plant Cell DOI: 10.1102/tpc.109.072272

Carlsbecker A, Helariutta Y (2005) Phloem and xylem 
specification: pieces of the puzzle emerge. Curr Opin Plant Biol 8: 512-517

Demura T, Fukuda H (2007) Transcriptional regulation in wood formation. Trends Plant Sci 12: 64-70

Demura, T, Tashiro G, Horiguchi G, Kishimoto N, Kubo M, Matsuoka N, Minami A, Nagata-Hiwatashi M, Nakamura K, Okamura Y, Sassa N, Suzuki S, Yazaki J, Kikuchi S, Fukuda H (2002) Visualization by comprehensive microarray analysis of gene expression programs during transdifferentiation of mesophyll cells into xylem cells. Proc Natl Acad Sci USA 99: 15794-15799

Fukuda H (1996) XYLOGENESIS: INITIATION, PROGRESSION, AND CELL DEATH. Annu Rev Plant Physiol Plant Mol Biol 47: 299-325

Fukuda H (2004) Signals that control plant vascular cell differentiation. Nat Rev Mol Cell Biol 5: 379-391

Ko JH, Kim WC, Han KH (2009) Ectopic expression of MYB46 identifies transcriptional regulatory genes involved in secondary wall biosynthesis in Arabidopsis. Plant J 60: 649-665

Ko JH, Yang SH, Park AH, Lerouxel O, Han KH (2007) ANAC012, a member of the plant-specific NAC transcription factor family, negatively regulates xylary fiber development in Arabidopsis thaliana. Plant J 50: 1035-1048

Kubo M, Udagawa M, Nishikubo N, Horiguchi G, Yamaguchi M, Ito J, Mimura T, Fukuda H, Demura T (2005) Transcription switches for protoxylem and metaxylem vessel formation. Genes Dev 19: 1855-1860

McCarthy RL, Zhong R, Ye ZH (2009) MYB83 is a direct target of SND1 and acts redundantly with MYB46 in the regulation of secondary cell wall biosynthesis in Arabidopsis. Plant Cell Physiol 50: 1950-1964

Mitsuda N, Iwase A, Yamamoto H, Yoshida M, Seki M, Shinozaki K, Ohme-Takagi M (2007) NAC transcription factors, NST1 and NST3, are key regulators of the formation of secondary walls in woody tissues of Arabidopsis. Plant Cell 19: 270-280

Mitsuda N, Ohme-Takagi M (2008) NAC transcription factors NST1 and NST3 regulate pod shattering in a partially redundant manner by promoting secondary wall formation after the establishment of tissue identity. Plant J 56: 768-778

Mitsuda N, Seki M, Shinozaki K, Ohme-Takagi M (2005) The NAC transcription factors NST1 and NST2 of Arabidopsis regulate secondary wall thickenings and are required for anther dehiscence. Plant Cell 17: 2993-3006

Nakano Y, Nishikubo N, Goué N, Ohtani M, Yamaguchi M, Katayama Y, Kajita S, Demura T (2010) MYB transcription factors orchestrating the developmental program of xylem vessels in Arabidopsis roots. Plant Biotechnol 27: 267-272

Olsen AN, Ernst HA, Leggio LL, Skriver K (2005) NAC transcription factors: structurally distinct, functionally diverse. Trends Plant Sci 10: 79-87

Ooka H, Satoh K, Doi K, Nagata T, Otomo Y et al. (2003) Comprehensive analysis of NAC family genes in Oryza sativa and Arabidopsis thaliana. DNA Res 10: 239-247

Soyano T, Thitamadee S, Machida Y, Chua NH (2008)
ASYMMETRIC LEAVES2-LIKE19/LATERAL ORGAN BOUNDARIES DOMAIN30 and ASL20/LBD18 regulate tracheary element differentiation in Arabidopsis. Plant Cell 20: 3359-3373

Turner S, Gallois P, Brown D (2007) Tracheary element differentiation. Annu Rev Plant Biol 58: 407-433

Willemsen V, Bauch M, Bennett T, Campilho A, Wolkenfelt H, Xu J, Haseloff J, Scheres B (2008) The NAC domain transcription factors FEZ and SOMBRERO control the orientation of cell division plane in Arabidopsis root stem cells. Dev Cell 15: 913-922

Yamaguchi M, Goúe N, Igarashi H, Ohtani M, Nakano Y, Mortimer JC, Nishikubo N, Kubo M, Katayama Y, Kakegawa K, Dupree P, Demura T (2010a) VASCULAR-RELATED NAC-DOMAIN6 (VDN6) and VND7 effectively induce transdifferentiation into under control of an induction system. Plant Physiol DOI: 10.1104/pp.110.154013

Yamaguchi M, Kubo M, Fukuda H, Demura T (2008) VASCULAR-RELATED NAC-DOMAIN7 is involved in the differentiation of all types of xylem vessels in Arabidopsis roots and shoots. Plant J 55: 652-664

Yamaguchi M, Ohtani M, Mitsuda N, Kubo M, Ohme-Takagi M, Fukuda H, Demura T (2010b) VND-INTERACTING2, a NAC domain transcription factor, negatively regulates xylem vessel formation in Arabidopsis. Plant Cell DOI: 10.1105/ tpc. 108.064048

Zhao C, Avci U, Grant EH, Haigler CH, Beers EP (2008) XND1, a member of the NAC domain family in Arabidopsis thaliana, negatively regulates lignocellulose synthesis and programmed cell death in xylem. Plant J 53: 425-436

Zhao C, Craig JC, Petzold HE, Dickerman AW, Beers EP (2005) The xylem and phloem transcriptomes from secondary tissues of the Arabidopsis root-hypocotyl. Plant Physiol 138: 803-818

Zhong R, Demura T, Ye ZH (2006) SND1, a NAC domain transcription factor, is a key regulator of secondary wall synthesis in fibers of Arabidopsis. Plant Cell 18: 3158-3170

Zhong R, Lee C, Ye ZH (2010) Functional Characterization of Poplar Wood-Associated NAC Domain Transcription Factors. Plant Physiol 152: 1044-1055

Zhong R, Lee C, Zhou J, McCarthy RL, Ye ZH (2008) A battery of transcription factors involved in the regulation of secondary cell wall biosynthesis in Arabidopsis. Plant Cell 20: 2763-2782

Zhong R, Richardson EA, Ye ZH (2007a) The MYB46 transcription factor is a direct target of SND1 and regulates secondary wall biosynthesis in Arabidopsis. Plant Cell 19: 2776-2792

Zhong R, Richardson EA, Ye ZH (2007b) Two NAC domain transcription factors, SND1 and NST1, function redundantly in regulation of secondary wall synthesis in fibers of Arabidopsis. Planta 225: 1603-1611.

Zhou J, Lee C, Zhong R, Ye ZH (2009) MYB58 and MYB63 are transcriptional activators of the lignin biosynthetic pathway during secondary cell wall formation in Arabidopsis. Plant Cell 21: 248-266 